\title{
Individual differences in anticipatory mu rhythm modulation are associated with executive function and processing speed
}

\author{
Staci Meredith Weiss ${ }^{1} \cdot$ Rebecca N. Laconi ${ }^{1} \cdot$ Peter J. Marshall ${ }^{1}$ \\ Published online: 13 August 2020 \\ (C) The Psychonomic Society, Inc. 2020
}

\begin{abstract}
There is increasing interest in the role of brain oscillations in the regulation and control of behavior. The current study examined the relations between specific cognitive abilities and changes in brain oscillatory activity during anticipation of, and in response to, tactile stimulation of the hand. The oscillation of interest was the sensorimotor mu rhythm $(8-14 \mathrm{~Hz})$ at central electrode sites. The electroencephalogram (EEG) was recorded during a task in which a visuospatial cue directed adults $(\mathrm{N}=40)$ that a tactile stimulus would be delivered to their left or right hand. Lateralized changes in mu power following tactile stimulation were associated with reaction time to the tactile stimulus. The extent of a contralateral anticipatory reduction in mu power during the $500 \mathrm{~ms}$ before the tactile stimulus was associated with performance on a separate processing speed task. Changes in ipsilateral mu power during anticipation of the tactile stimulus were associated with performance on a flanker task and were marginally correlated with performance on a card sort task. Regression analyses further indicated the specificity of these relations to anticipatory changes in mu power. In summary, mu rhythm modulation during anticipation of tactile stimulation to a specific bodily location was related to a broad measure of processing speed and to variability in the broader ability to regulate behavior in a goal-directed manner. Implications are discussed in terms of the foundational role of anticipatory attention in cognitive processes and the utility of selective attention to the body as an index of attentional control more broadly.
\end{abstract}

Keywords Attention $\cdot$ Regulation $\cdot$ Executive function $\cdot$ Tactile $\cdot$ Alpha $\cdot$ Mu rhythm

\section{Introduction}

Neuroimaging studies have shed light on how anticipation of an expected stimulus is associated with specific patterns of cortical activity, including activation of primary sensory cortices (Corbetta et al., 2000; Nobre \& van Ede, 2018). Such anticipatory responses reflect the deployment of attention in a proactive, selective manner in expectation of an upcoming sensation. In turn, it has been suggested that the ability to direct attention to sensations in a specific and selective manner may foster control of attention and action more broadly (Jha, Krompinger, \& Baime, 2007). Individual differences in cortical activity during anticipation may explain critical variability in behavioral responses (Buzsaki, 2006; Lopes da Silva, 2013).

Staci Meredith Weiss

smeredithweiss@gmail.com

1 Department of Psychology, Temple University, 1701 N. 13th Street, Philadelphia, PA 19122, USA
In experimental settings, when the presentation of a visual, auditory, or tactile target is preceded by a stimulus-relevant cue, participants demonstrate more accurate responses and faster reaction times than when a stimulus is presented without a preparatory cue (Posner, 1980; Frey et al., 2015). This established finding suggests that prior to stimulus presentation, deployment of attention in a selective, focused manner improves stimulus processing (Nobre \& van Ede, 2018). Exploiting the temporal precision of the electroencephalogram (EEG), we can eavesdrop on rhythmic neural responses that change during anticipation of, and in response to, a stimulus (Cheyne et al., 2003; Engel, Fries, \& Singer, 2001).

Prior research investigating sensory anticipation, reaction time, and higher-order control of action has primarily employed stimuli in the visual and auditory modalities (McKinney \& Euler, 2019; Zanto \& Gazzaley, 2009), with much less empirical work focused on how attention in the tactile modality contributes to variance in adapting behavior to task demands. We suggest that attention to tactile stimuli that are presented on the body surface may differ fundamentally from attention to visual and auditory stimuli presented in extrapersonal space (Holmes \& Spence, 2004; Bremner, 
Holmes \& Spence, 2012; Serino, 2019). Somatosensory processing is distinct from auditory and visual processing, because it inherently consists of self-relevant information and is integrated with the body schema (Haggard, Taylor-Clarke, \& Kennett, 2003; Holmes \& Spence, 2004). Anticipation of tactile stimuli reallocates attention from passively monitoring the global surface of the body to scanning ongoing sensation at a specific, localized area on the body surface (GomezRamirez, Hysaj, \& Niebur, 2016). Orienting, anticipating, and reacting to specific tactile sensations is contextualized by the postural, functional, and lateral dimensions of input (Longo, Azañón, \& Haggard, 2010; Tamè, Azañón, \& Longo, 2019). In particular, the lateralized nature of somatosensory processing is critical to our index of anticipation (Tamè et al., 2019).

Anticipation of tactile stimuli reallocates attention from passively monitoring the global surface of the body to scanning ongoing sensation at a specific, localized area on the body surface (Gomez-Ramirez, Hysaj, \& Niebur, 2016). There also are several strengths of employing somatosensory stimuli: (i) Compared with the visual modality, tactile attention is not complicated by factors, such as ocular shifts or visual preferences; (ii) Neural indices of anticipation of touch are readily measurable through EEG recordings from electrodes overlying somatosensory cortex (Anderson and Ding, 2011; Haegens et al., 2011); (iii) The ability to focus attention to a body part and concomitant brain responses in expectation of touch may be amenable to change and enhancement via interventions that target the training of attention to the body and interoceptive activity (Allen et al., 2012; Jones et al., 2010; Zeidan et al., 2010).

The current study investigates whether individual differences in the patterning of EEG oscillations immediately before and following delivery of a cued tactile stimulus are related to meaningful variability in behavior. Our primary goal was to examine individual differences in oscillatory neural responses during anticipation of a tactile stimulus and in response to that stimulus. A preparatory cue was presented in the visual modality, allowing temporal and spatial differentiation of neural activity specific to stimulus anticipation (over sensory cortex relevant to the tactile target) from sustained neural and behavioral responses elicited by the visual cue.

\section{The active role of alpha oscillations in anticipation and perception}

Expectation-related changes in electrophysiological responses have provided a useful tool for studying selective enhancement of sensory signals and concomitant changes in behavior consistent with selective attention, or the voluntary control of the attentional spotlight (Posner, 1980). Oscillations in the alpha band (around $8-14 \mathrm{~Hz}$ in adults) have been identified as a correlate, gate, and predictor of behavioral responses and cognitive functioning (Klimesch et al., 1998; Nobre \& van Ede, 2018; Zanto \& Gazzaley, 2009). Decreases in alpha power during stimulus anticipation (before stimulus onset, following a stimulusrelevant cue) are observed at electrode sites selective to the contralateral stimulus-relevant sensory cortex, with greater decreases generally associated with improved perceptual report, behavioral responses, and measures of cognitive skills, such as working memory (Murphy, Foxe, \& Molholm, 2016; Zanto and Gazzaley, 2009). In contrast, increases in alpha power responses evoked by presentation of a target stimulus account for additional variability in behavioral responses to the target (Thut et al., 2006; Mazaheri et al., 2009; Van Diepen et al., 2015).

EEG oscillations are thought to arise from fluctuations in the polarity of cortical tissue that reflect the shifting, homeostatic balance of postsynaptic potentials released by assemblies of excitatory pyramidal cells and inhibitory interneurons (Lopes da Silva, 2013; Cohen, 2014). The delivery (or mere expectation) of a stimulus is thought to disrupt the ongoing synchronized firing rate of postsynaptic potentials that generate rhythmic activity, eliciting an event-related desynchronization (ERD) in the oscillatory signal (Haegens et al. 2011; Lopes da Silva, 2013). Changes in amplitude and phase of oscillations evoked by a discrete event can be quantified via event-related spectral perturbation (ERSP), using sinusoidal wavelets to estimate shifts in amplitude and phase of EEG oscillations (Makeig \& Delorme, 2004; Klimesch et al., 1998).

Contemporary accounts of "top-down" or attention-related modulation of alpha-range oscillatory activity rest upon the inhibition-timing hypothesis (Klimesch, Sauseng, \& Hanslmayr, 2007). This hypothesis proposes that when a stimulus disrupts a default state of rest or inattention, the inhibition of global neural activity allows a reallocation of neurophysiological resources, which are diverted to local processing of the new or expected stimulus. Widespread increases in alpha power from baseline may therefore reflect inhibited sampling of irrelevant sensory events, with enhanced sampling of events (signaled by a reduction in alpha power) in areas of cortex relevant to stimulus processing (Klimesch, Sauseng, \& Hanslmayr, 2007; Mazaheri et al., 2009; Schroeder \& Lakatos, 2009). As such, concomitant global increases and local decreases in oscillatory EEG power are associated with cognitive processes and mental states, such as event expectation, inhibition of attention, and perceptual awareness of a stimulus (Frey et al., 2015; Schroeder \& Lakatos, 2009; Thut et al., 2006).

In studies of how alpha-range rhythms change in anticipation of, and in response to, a discrete event, participants are typically presented with a cue that orients them to a feature of the target stimulus. In the widely used Posner 
paradigm, a spatial cue indicates whether a target visual stimulus will be presented in the participant's right or left visual field (Posner, 1980; Foxe \& Snyder, 2011). During the interval following the cue, but before the predicted onset of a visual stimulus, anticipatory desynchronization of alpha-range oscillations is observed over contralateral visual cortex, manifesting as a decrease in alpha power relative to baseline at contralateral occipital electrodes (Foxe \& Snyder, 2011; Sauseng et al., 2005; Thut et al., 2006; Nobre \& van Ede, 2018).

EEG studies of anticipation in the tactile modality have focused on the sensorimotor mu rhythm, which exhibits dissociable properties from the posterior alpha rhythm that is more closely associated with visual processing (Yin, Liu \& Ding, 2016). While visual alpha is typically recorded from occipital electrodes, the mu rhythm is prominent over central electrode sites (Jones et al., 2010; Pfurtscheller, 1989). Unlike alpha rhythms over other regions, sensorimotor mu oscillations exhibit a characteristic Rolandic shape when synchronized and change in response to tactile features of a stimulus, even when visual or motor stimuli remain the same (Coll et al., 2017). Expectation of a tactile stimulus elicits changes in the mu rhythm that exhibit a somatotopic pattern (Anderson \& Ding, 2011; Jones et al., 2010), in accord with the homuncular organization of somatosensory cortex (Penfield \& Boldrey, 1937). For instance, Jones et al., (2010) demonstrated that anticipatory $\mathrm{mu}$ responses are lateralized when a spatial cue (pointing left or right) directs participants to attend to one of their hands, which subsequently received a tactile stimulus. Consistent with other adult EEG studies involving expectation of touch (Shen et al., 2018; Anderson \& Ding, 2011; van Ede, Jensen, \& Maris, 2010), an anticipatory reduction of mu power was localized to central sites contralateral to the direction of the cue (Jones et al., 2010). These studies and other related investigations have focused on anticipatory changes in the mu rhythm as predictors and correlates of variability in behavioral responses that are directly relevant to the processing of tactile stimuli (Haegens, Luther, \& Jensen, 2012; van Ede, de Lange, \& Maris, 2014). Although contralateral changes in the mu rhythm have been the main focus of EEG work on tactile anticipation, mu responses at ipsilateral central sites are also of interest. When a distracting tactile sensation is presented simultaneously to the uncued hand, ipsilateral increases in mu power (event-related synchronization; ERS) occur, possibly indexing the suppression of task-irrelevant stimulation (Haegens, Luther, \& Jensen, 2012; van Ede, de Lange, \& Maris, 2014). This inhibitory function ascribed to alpharange oscillatory activity has served to explain the association between anticipatory ipsilateral increases in alpha power with the accuracy and speed of behavioral responses, with this effect being found across sensory modalities (Samaha and Postle 2015; van Diepen et al. 2015).

\section{Modulation of Mu power and tactile processing}

Across the auditory, visual, and tactile modalities, both contralateral reductions and ipsilateral increases in alpha power that are apparent during anticipation of a target stimulus and to the presentation of that stimulus have been correlated with behavioral responses to the target (Thut et al., 2006; van Ede et al., 2014; Frey et al., 2015). In the tactile modality, the relationship between changes in mu power and behavioral indicators of tactile processing differs depending on the strength and salience of the expected tactile stimulation, as well as the load on tactile attention (Haegens et al., 2012; Gomez-Ramirez, Hysaj, \& Niebur, 2016). When a reliable spatial cue directs participants to expect tactile stimulation at the cued location, the magnitude of anticipatory mu desynchronization at contralateral central electrode sites is linearly and inversely associated with accuracy of stimulus detection (Anderson \& Ding, 2011; Haegens, Handel, \& Jensen, 2011; Jones et al., 2010). Van Ede et al. (2012) examined anticipatory and post-stimulus mu power to parse their relative contributions to behavioral indicators of tactile processing. The extent of anticipatory mu desynchronization was significantly related to the accuracy of participant's tactile judgements, while both the magnitude of anticipatory mu desynchronization and post-stimulus mu increases in mu power were associated with participants' reaction times to the stimulus.

In contrast to the set of studies which support a linear association of mu power and behavioral report of tactile stimuli, Wittenberg, Baumgarten, Schnitzler, \& Lange, (2018) identified a quadratic relation between accurate detection of sequential tactile stimuli and anticipatory mu power in the ipsilateral hemisphere only. In an investigation using MEG, LinkenkaerHansen et al. (2004) reported a quadratic relationship between participant's ability to detect a weak tactile stimulus and their anticipatory contralateral mu oscillatory activity. Similarly, Zhang and Ding (2010) found that participants with intermediate levels of anticipatory contralateral mu ERD had the highest hit rates in detecting a subthreshold tactile stimulus, which was psychophysiological-titrated for each participant. To address inconsistencies in the literature associating oscillatory mu activity with task-specific indicators of tactile processing, we note the potential importance of subtle differences in task demands (Gomez-Ramirez et al., 2016).

Differences between ipsilateral and contralateral mu power decreased when the probability of spatial cue accuracy was manipulated - increasing uncertainty and decreasing prediction (Haegens et al., 2011). Particularly when tactile attention is divided between multiple bodily locations, it appears that anticipation of tactile stimulation involves both ipsilateral and 
contralateral somatosensory cortices (Haegens et al., 2011; Haegens, Luther, \& Jensen, 2012). Similar to the finding that increases in ipsilateral anticipatory alpha power in the visual modality account for variability in stimulus response (Thut et al., 2006; Frey et al., 2015), we understand anticipatory ipsilateral mu power as an index of selective, inhibited, sampling of sensory events when tactile attention is under load (Frey et al., 2015; Tamè et al., 2016).

\section{Current investigation: modulation of mu power during selective attention to the hands}

One suggestion arising from work linking anticipatory neural responses to basic sensory responses is the proposition that "low-level" indicators of attentional processing reciprocally influence, gate, and cascade into individual level differences in the "higher-order" ability to control behavioral responses (Engel, Fries \& Singer, 2001; Gazzaley and Nobre, 2012; Sadaghiani \& Kleinschmidt, 2016). In the current study, individual differences in oscillatory EEG responses were assessed during anticipation of a tactile stimulus and in response to that stimulus. Subject-specific changes in oscillatory activity were investigated in relation to 1) reaction time to respond to the tactile stimulus, 2) general processing speed and receptive language abilities, and 3) executive function abilities, or the constellation of skills involved in the regulation of behavior.

We are interested in utilizing changes in mu rhythm modulation to understand individual variation in the cognitive and perceptual processes which occur during selective attention to the right or left hand, before tactile stimulation, following a directional visual cue (anticipation) and following tactile stimulation (post-stimulus). We suggest that executive function, defined by the planning, regulating, and monitoring of goaldirected behavior may partly be a manifestation of individual differences in how adults use information in their environment, influencing their anticipation of upcoming sensory events and adjustment of behavior to such expectancies. Further developmental inquiries can unpack the direction of this association: it may be that anticipation is a foundational aspect of cognitive development, which cascades into variability in stimulus response and attention deployment, as indexed by trial-by-trial behavioral responses and executive function (Garon et al., 2008; Weiss et al., 2018). Tactile anticipation is advanced here as an interesting index of individual differences, because somatosensory attention is not directly assessed by executive function tasks. Furthermore, alpha lateralization captures a wider range of interindividual variability in neural and behavioral responses, relative to intraindividual variability of alpha peak or power across trials (Jones et al., 2010; Baumgarten et al., 2019).

There is some precedent in the literature linking anticipatory EEG responses with indicators of cognitive performance (Klimesch, 1999). Many of these studies have utilized task- specific outcomes, such as reaction time, as indicators of higher-order executive abilities. McKinney and Euler (2019) employed a unimodal visual selective attention paradigm to identify the association of alpha modulation higher fluid intelligence but was mediated by reaction time and temporal anticipation (McKinney \& Euler, 2019). Open questions remain regarding whether these anticipatory processes serve the same functions in a multimodal-cued paradigm in adults. Singletrial reaction time index intraindividual variability and individual differences in general speed of processing, which contribute to- but are distinct from-executive function skills (Willoughby et al., 2018; McKinney, Euler \& Butner, 2020)

Prior studies have linked variation in anticipatory oscillatory responses during expectation of a visual stimulus to multiple correlates of inter- and intra-individual variation in attention deployment and processing speed, including working memory (Zanto \& Gazzaley, 2009), task effort (Myers et al., 2014), time-on-task (Rungratsameetaweemana et al., 2019), stimulus reaction time (Sauseng et al., 2005), and stimulus detection or hit rate (Thut et al., 2006; Samaha and Postle 2015; van Diepen et al. 2019). Executive function abilities are proposed to serve as a bridge between reaction to individual stimuli and higher-order self-regulation of behavior (Nigg, 2017). The tasks employed by the standardized NIH Cognitive Toolbox battery to measure executive function are highly linked to an action-oriented definition of its role in cognitive, emphasizing behavioral inhibition (action suppression) and cognitive flexibility (action adjustment) aspects, rather than the separable component of working memory capacity (Miyake and Freidman, 2012) function abilities can be indexed by "marker" tasks, which involve the coordination of competing attentional priorities.

To index behavioral inhibition, the Flanker task is administered, in which participants indicate the direction of a central arrow that was presented between distractor or "flanker" arrows. The direction of arrows is randomized by trial, such that the flanking arrows are alternatively congruent or incongruent with the target central arrow. To index cognitive flexibility, the Dimensional Change Card Sort (DCCS) was administered. The DCCS instructs participants to match the central test stimuli with one of two lateralized target stimuli by shape or color, such that participants on the first block of the task sort the cards by one dimension, and on the next block sort the same cards by the other dimension. Compared with the DCCS, the Flanker is considered a more specific "conflict" task: it primarily indexes inhibitory control (Diamond, 2013), such that incongruent conditions are associated with slower reaction times, which is explained as participants reconciling the conflict between the target and distractor.

Based on prior studies, we expected to find a significant association between anticipatory mu modulation and reaction time following a delay in reporting the number of tactile stimuli detected (Haegens et al., 2011; Gomez-Ramirez et al., 
2016; Jones et al., 2010; van Ede et al., 2012). Considering critical role of expectation in multiple theoretical accounts of cognition control and perceptual decision-making (de Lange, Heilbron, \& Kok, 2018; Summerfield and De Lange, 2014), we were particularly interested in examining whether the magnitude of mu rhythm modulation elicited in anticipation of and in response to tactile stimulation accounted for significant individual variance in executive function skills (unrelated to the tactile detection task). The attentional allocation and inhibition of attention roles of anticipatory alpha power may be differentially associated with different aspects of executive function, specifically with adult ipsilateral mu power suggested as a potential correlate of the Flanker, an index of inhibition. Correlating neural modulation specific to tactile expectation with broader variability in cognitive functioning is fairly novel (as opposed to tactile perception; see Spitzer \& Blankenburg, 2011; Ricciardi et al., 2006), but complements accumulating evidence for the critical role of anticipatory lateralization of alpha responses as an index of broader behavioral outcomes in the visual modality (Van Diepen, Foxe, \& Mazaheri, 2019; Van der Lubbe, \& Utzerath, 2013; McKinney \& Euler, 2019). Separate regression models on contralateral and ipsilateral mu power address whether variability related to the functional allocation or inhibition of attention. This approach aids interpretability of the widely used lateralization index (Haegens et al., 2011), which will be tested in anticipation and in response to tactile stimulus delivery. Furthermore, pre-stimulus and post-sitmulus mu power is examined in relation to individual differences in cognitive and behavioral outcomes.

\section{Methods}

\section{Participants}

Fifty undergraduate students received course credit in return for participation. Informed consent was obtained from all participants. Data from four participants were excluded from analyses due to technical problems during EEG collection. An additional six participants were excluded due to excessive motor and/or eye movement artifacts that contaminated more than $25 \%$ of trials. The final analyzed sample comprised 40 participants (mean age $=21.24$ years; standard deviation [SD] $=3.85$ ). All participants were right-handed according to the Oldfield Handedness questionnaire (Oldfield, 1971), were neurologically healthy, and had normal or corrected vision. Participants were fitted with an EEG cap and tactile stimulators, seated at a table facing a computer screen, and instructed to rest their hands on their lap, under the table and out of sight.

\section{Somatosensory selective attention task}

Participants were instructed to prepare for tactile stimulation to the middle finger of the hand indicated by the direction of the arrow and to indicate how many tactile stimuli they detected (one or two) by pressing a foot pedal once or twice. The specific sequence of visual stimuli in each trial comprised a fixation cross for $500 \mathrm{~ms}$, followed by the arrow cue for 2,250 ms, followed by a response screen that read "Copy with Your Foot!" (Fig. 1). Participants were directed to respond immediately following the onset of the response screen. The direction of the arrow was randomized, with an equal number (100) of left and right trials. Prior to the experimental trials, five practice trials were presented to ensure that participants distinguished between the single and double tactile stimuli. Two tactile stimuli were delivered in rapid succession ("double stimuli") on 20 of the 100 trials to each hand. The foot used to respond to stimuli was counterbalanced (right or left) between participants. Reaction time for each participant was computed as the time from the onset of the response screen to the onset of a foot pedal press.

Tactile stimuli were delivered to the distal tip of the left and right middle fingers using an inflatable membrane (10-mm diameter; MEG Services International, Coquitlam, BC) mounted in a plastic casing and secured with a finger clip. The membrane was inflated by a short burst of compressed air delivered via flexible polyurethane tubing (3-m length, 3.2$\mathrm{mm}$ outer diameter). The compressed air delivery was controlled by STIM stimulus presentation software in combination with a pneumatic stimulator unit (both from James Long Company, Caroga Lake, NY) and an adjustable regulator that restricted the airflow to 60 psi. To generate each tactile stimulus, the STIM software delivered a 10-ms trigger that served to open and close a solenoid in the pneumatic stimulator. The time zero point in the EEG analyses was adjusted to reflect the onset of membrane expansion, which started $15 \mathrm{~ms}$ after trigger onset and peaked $35 \mathrm{~ms}$ later, with a total duration of membrane movement of around $100 \mathrm{~ms}$.

\section{EEG collection}

EEG was recorded at $512 \mathrm{~Hz}$ using a stretch cap (ANT Neuro, Berlin) with electrodes placed at Fp1, Fpz, Fp2, F3, Fz, F4, F7, FC6, FC1, FC2, FC5, F8, Fz, C3, Cz, C4, CP1, CP2, CP5, CP6, T7, T8, P3, Pz, POz, P4, P7, P8, O1, Oz, O2, and the left and right mastoids. The electrooculogram (EOG) was recorded from above and below the orbital rim of the left eye. Conducting gel was used and scalp electrode impedances were kept under $25 \mathrm{k} \Omega$ (values were typically lower). EEG channels were collected referenced to the vertex $(\mathrm{Cz})$ and were re-referenced offline to an average mastoids reference prior to further analysis. EEG and EOG signals were amplified using optically isolated, high input impedance ( $>1 \mathrm{G} \Omega$ ) custom 


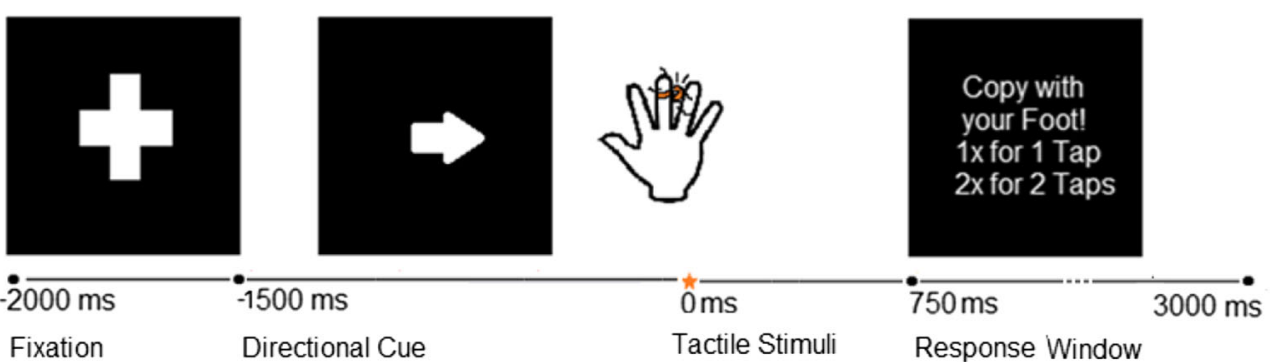

Fig. 1. Trial structure. A fixation point was displayed for $500 \mathrm{~ms}$, followed by an arrow (directional cue) displayed continuously for $2250 \mathrm{~ms}$, with the delivery of the tactile stimulus occurring $1,500 \mathrm{~ms}$ later (at $0 \mathrm{~ms}$ ). The response prompt was displayed at $750 \mathrm{~ms}$ after tactile stimulus onset

bioamplifiers (SA Instrumentation, San Diego) and digitized using a 16-bit $\mathrm{A} / \mathrm{D}$ converter $( \pm 2.5 \mathrm{~V}$ input range). For the EEG channels, bioamplifier gain was 4,000 and the hardware filter (12 dB/octave rolloff) settings were $0.1 \mathrm{~Hz}$ (high-pass) and $100 \mathrm{~Hz}$ (low-pass).

\section{EEG processing}

Initial processing of the data utilized the EEG Analysis System (James Long Company) followed by analysis using the EEGLAB toolbox (Delorme \& Makeig, 2004) version 13.5.4b implemented in MATLAB. Following standard guidelines for EEG analyses (Bigdely-Shamlo et al., 2015), high $(0.01 \mathrm{~Hz})$ and low-pass $(40 \mathrm{~Hz})$ filters were employed and visual inspection of the EEG signal was then used to reject epochs containing excessive artifact. Independent component analysis was used to clear the EEG data of ocular and muscle artifact (Hoffmann \& Falkenstein, 2008). There was no significant difference in the number of usable trials between the left and right cued conditions ( $p=0.81)$ or in relation to behavioral measures. Of 80 trials, the mean number of artifactfree trials per condition was $69(\mathrm{SD}=5.62)$.

For each single-pulse trial with a correct behavioral response, a 2,500-ms epoch was extracted that began 2,000 ms before the onset of the tactile stimulus and extended $500 \mathrm{~ms}$ after tactile stimulus onset. Spectral power over this epoch was estimated using Gaussian-tapered Morlet wavelets. Changes in power were computed as event-related spectral perturbation (ERSP) following visual cue presentation (i.e., $-1,500$ to $500 \mathrm{~ms}$ following tactile stimulus presentation) relative to a $500 \mathrm{~ms}$ baseline preceding the visual cue (i.e., $-2,000$ to $-1,500 \mathrm{~ms}$ before tactile stimulation onset). For statistical analyses, a key variable was mu ERSP at electrodes $\mathrm{C} 3$ and $\mathrm{C} 4$. The specific focus on central electrodes was based on the well-documented reactivity of the mu rhythm over the central region during anticipation of, and responding to, tactile stimulation (Anderson and Ding, 2011; Haegens et al., 2011; Shen et al., 2018).

An individual-specific adaptive mean approach was employed to identify the mu frequency range at $\mathrm{C} 3$ and $\mathrm{C} 4$ in each participant. The individual mu peak frequency detection identified the largest local maximum within the 7$14 \mathrm{~Hz}$ range (Haegens et al., 2014). The mu peak selection was conducted in the $1,000 \mathrm{~ms}$ before delivery of the tactile stimulus $(-1,000$ to $0 \mathrm{~ms})$ to prevent contamination by changes evoked by the visual cue that were evident from $-1,500$ to $-1,000 \mathrm{~ms}$. Temporal window selection for pre- and poststimulus windows employed an individual-specific and group-mean approach, testing in 10-ms increments for significant differences in mu power relative to baseline. There were no significant differences evident in mu power or relations observed based on temporal approach, so standardized windows were employed across participants. Mu ERSP during tactile anticipation was examined in the 500-ms window before onset of the tactile stimulus $(0 \mathrm{~ms}$, when the membrane began to move), within a subject-specific frequency range ( $\mathrm{mu}$ peak frequency $\pm 2 \mathrm{~Hz}$ ).

$\mathrm{Mu}$ ERSP in response to the tactile stimulus was computed for the period from $20 \mathrm{~ms}$ to the $270 \mathrm{~ms}$ following tactile stimulus onset, using the same subject-specific frequency range. The onset of the post-stimulus window corresponded to the peak of membrane expansion.

\section{Extracting anticipatory Mu response relative to baseline}

Following the methodological recommendations of Cuevas et al. (2014), we initially calculated absolute (i.e., without baseline correction) power values to confirm that contralateral and ipsilateral mu power prior in the 500 -ms period immediately preceding and the $250-\mathrm{ms}$ period following the tactile stimulus differed from power in the same frequency band (8$14 \mathrm{~Hz}$ ) during the baseline period $(-2,000$ to $-1,500 \mathrm{~ms})$.

Pairwise comparisons indicated that at the left central electrode site $(\mathrm{C} 3)$, mu power during anticipation of stimulation to the right hand was significantly reduced compared with the baseline epoch $(p<0.001)$. Differences in mu power between baseline and the anticipatory epoch at ipsilateral central electrode sites were apparent and were but only trended toward being significantly different from baseline $(p<0.10)$. At the right central electrode site $(\mathrm{C} 4)$, mu power during anticipation of stimulation to the left hand $(p<0.001)$ and the right hand 
$(p<0.01)$ was significantly lower than during the baseline epoch.

Further pairwise comparisons indicated that at the left central electrode site (C3), mu power in response to tactile stimulation of the right hand and the left hand was significantly greater than during the baseline epoch (both $p<0.001$ ). At the right central electrode site $(\mathrm{C} 4)$, mu power in response to stimulation of the left hand and the left hand was significantly greater than the baseline epoch (both $p<0.001$ ).

Given the confirmation of significant contralateral decreases in absolute mu power during anticipation (relative to baseline) and contralateral increases in absolute mu power in response to stimulation, the analyses described below use baseline-corrected ERSP values.

\section{Subject-specific identification of anticipatory Mu ERSP}

The envelope of the amplitude-modulated signal was computed via the Hilbert transform ("Hilbert" function in Matlab), which discards phase information and reveals oscillatory power fluctuations over time. A subject-specific approach to identifying the peak frequency of the mu rhythm was used, with a peak quantified in $\mathrm{R}$ as the largest local maximum within the 8-14 $\mathrm{Hz}$ range. This value was extracted from the output of spectral plots of individual participants for electrodes $\mathrm{C} 3$ and $\mathrm{C} 4$, for each condition (right/left). The mean mu peak frequency was $10.1 \mathrm{~Hz}$ with a between-subject SD of $2.1 \mathrm{~Hz}$ (compared with a within-subject SD of $0.8 \mathrm{~Hz}$ ), and the median was $10.4 \mathrm{~Hz}$. Mu peak frequency was not related to reaction time, hit rate, or any of the cognitive measures.

\section{Behavioral measures}

Following the tactile task and removal of the EEG cap, four tasks from the NIH Cognition Toolbox (for details, see Zelazo et al., 2013) were administered: the Flanker task and the Card Sort task measured aspects of executive function; a Processing Speed task required participants to evaluate rapidly whether two images were identical or not; and a picture vocabulary task measured Receptive Language. For all four tasks, tstandardized test scores were used (standardized around a population mean of 100) as provided by the NIH Cognitive Toolbox.

The dimensional change Card Sort task indexes cognitive flexibility and attention shifting facets of executive function (Zelazo, 2006). Participants were directed to select one of two test stimuli that matched the shape (truck or ball) or color (red or blue) of the target stimuli, as instructed by a verbal prompt that varied randomly between trials. In the Flanker task, participants were required to indicate the direction of an central arrow that was presented between distractor (flanker) arrows. The direction of arrows was randomized by trial, such that the flanker arrows were alternatively congruent or incongruent with the target central arrow. The Flanker task indexes response inhibition, selective attention, and conflict monitoring (Zelazo et al., 2013). Scores on the Card Sort and Flanker tasks reflected both accuracy and reaction time all participants, who correctly identified targets on $80 \%$ of trials, as is typical for neuropsychological healthy adults (Zelazo et al., 2013). Computed scores on of these tasks indicates an estimate of executive function ability (behavioral inhibition for Flanker, cognitive flexibility for Card Sort) by providing a value from 0 to 5 for each trial for optimal accuracy (5) and minimal, rapid reaction time (5); the final computed score is an average across trials that weighted the difficulty of each trial from $0-10$, with 0 being minimal from prior trial (preservation), and subsequent trials weighted when a nontarget image shifted in position from the prior trial $(+1)$, when a change in rule was employed (+2), when a change in rule and position created a conflict $(+3)$, and change in rule, image and position created an additional conflict $(+4)$. For more details, see the NIH Toolbox Guide to Interpreting Scores (Akshoomoff et al., 2013).

\section{Plan of analysis}

Statistical analyses were conducted in R (Howell, 2016) and focused on mu ERSP at the left and right central electrodes (C3 and C4) in anticipation of (anticipatory) and in response to a tactile stimulus (post-stimulus). Assumption checks determined that variables in the analyses were normally distributed. Table 1 shows descriptives for all relevant neural and behavioral variables. Repeated measures ANOVAs were conducted to examine differences in mu ERSP as a function of electrode and task condition (i.e., "Cue Direction"). Continuous relations between cognitive skills, reaction time, and anticipatory and post-stimulus mu modulation were evaluated using linear regression; significance is reported using two-tailed comparisons with FDR correction (Table 2).

\section{Results}

\section{Behavioral responses to single vs. double tactile pulses}

Across the entire sample $(\mathrm{N}=40)$, participants responded correctly to the single or double stimuli on $96.7 \%$ of trials. Hit rate was calculated as the number of correct responses over the total number of trials with artifact-free EEG data but was not submitted for further analysis, because it was not related to other study variables. Reaction time for each participant was calculated as the duration from the onset of the response screen until the initiation of the foot pedal press. Only single-stimulus trials with accurate responses were included in analyses of the EEG data to prevent contamination 
Table 1 Descriptive statistics for Mu ERSP and cognitive task scores

\begin{tabular}{llllllllll}
\hline & $\begin{array}{l}\text { Anticipatory } \\
\text { contralateral Mu } \\
\text { ERSP }\end{array}$ & $\begin{array}{l}\text { Anticipatory } \\
\text { ipsilateral Mu } \\
\text { ERSP }\end{array}$ & $\begin{array}{l}\text { Post-stimulus } \\
\text { contralateral Mu } \\
\text { ERSP }\end{array}$ & $\begin{array}{l}\text { Post-stimulus } \\
\text { ipsilateral Mu } \\
\text { ERSP }\end{array}$ & $\begin{array}{l}\text { Flanker Card } \\
\text { sort }\end{array}$ & $\begin{array}{l}\text { Working } \\
\text { memory }\end{array}$ & $\begin{array}{l}\text { Language Reaction } \\
\text { time (ms) }\end{array}$ \\
\hline Mean & -0.430 & -0.028 & 0.013 & 0.371 & 97.83 & 105.20 & 104.8 & 105.4 & 1.505 \\
Std. deviation & 0.797 & 0.803 & 1.543 & 1.414 & 16.65 & 14.00 & 16.04 & 13.06 & 0.169 \\
Skewness & 0.158 & -0.754 & -0.029 & 0.342 & 0.068 & -0.458 & 0.204 & -0.724 & 0.896 \\
Kurtosis & -0.030 & 1.219 & -0.676 & 0.381 & -0.557 & -0.095 & -0.894 & 0.957 & 0.338 \\
Range & 3.683 & 3.981 & 5.958 & 6.598 & 62.00 & 58.00 & 59.00 & 60.00 & 0.705 \\
\hline
\end{tabular}

of post-stimulus ERSP by double stimuli or error-related responses.

\section{Characterizing Mu ERSP}

Time-frequency plots (Fig. 2) show a clear mu rhythm (8-14 $\mathrm{Hz}$ ) desynchronization at the central electrode site (C3 or $\mathrm{C} 4)$ contralateral to cue direction. This desynchronization was particularly salient in the $500 \mathrm{~ms}$ immediately before tactile stimulus delivery. In contrast, there was minimal change in mu power over this period at the central electrode ipsilateral to the cue direction.

\section{Anticipatory Mu ERSP}

A repeated-measures ANOVA was conducted to compare anticipatory mean ERSP in the subject-specific frequency range (at $\pm 2 \mathrm{~Hz}$ from individual mu peak frequency) in the 500-ms window before tactile stimulation by electrode $(\mathrm{C} 3 / \mathrm{C} 4)$ and cue direction (left/right). No main effects were observed. A significant interaction was observed between cue direction and electrode, $\mathrm{F}(1,39)=25.757, p<0.001, \eta^{2 \mathrm{p}}=0.398$. As suggested by the ERSP scalp maps (Fig. 3), this interaction was driven by more negative ERSP (i.e., greater mu desynchronization) at the contralateral site than at the ipsilateral site. When stimulation was expected to the left hand, mu desynchronization was greater at $\mathrm{C} 4(\mathrm{M}=-0.461, \mathrm{SD}=$ $0.988)$ than at $\mathrm{C} 3(\mathrm{M}=-0.022, \mathrm{SD}=0.984, \mathrm{t}=3.246, p<$ $0.001, d=0.588)$. When stimulation was expected to the right hand, mu desynchronization was greater at C3 $(\mathrm{M}=-0.398$, $\mathrm{SD}=1.026)$ than at $\mathrm{C} 4(\mathrm{M}=-0.077, \mathrm{SD}=0.844, \mathrm{t}=-3.246$, $p=0.002, \mathrm{~d}=-0.513$ ) (Figs. 4 and 5).

\section{Post-stimulus Mu ERSP}

A repeated-measures ANOVA was conducted to compare mean $8-14 \mathrm{~Hz}$ ERSP in the 20 to $270 \mathrm{~ms}$ window by electrode (C3/C4) and cue direction (left/right). No main effects were

Table 2 Correlation matrix of study variables

\begin{tabular}{|c|c|c|c|c|c|c|c|c|}
\hline \multicolumn{2}{|c|}{$\begin{array}{l}\text { Contralateral anticipatory } \mathrm{Mu} \\
\text { ERSP }\end{array}$} & \multirow{2}{*}{$\begin{array}{l}\text { Ipsilateral } \\
\text { anticipatory } \mathrm{Mu} \\
\text { ERSP }\end{array}$} & \multirow{2}{*}{$\begin{array}{l}\text { Contralateral post- } \\
\text { stimulus Mu ERSP } \\
0.718^{* * *}\end{array}$} & \multirow{2}{*}{$\begin{array}{l}\text { Ipsilateral post- } \\
\text { stimulus Mu ERSP } \\
0.667 * * *\end{array}$} & \multirow{2}{*}{$\begin{array}{l}\text { Flanker } \\
(\mathrm{EF}) \\
-\end{array}$} & \multirow{2}{*}{$\begin{array}{l}\text { Card sort } \\
\text { (EF) } \\
-\end{array}$} & \multirow{2}{*}{$\begin{array}{l}\text { Processing } \\
\text { speed }\end{array}$} & \multirow{2}{*}{$\begin{array}{l}\text { Language } \\
--\end{array}$} \\
\hline $\begin{array}{l}\text { Contralateral } \\
\text { anticipatory } \mathrm{Mu} \\
\text { ERSP }\end{array}$ & - & & & & & & & \\
\hline $\begin{array}{l}\text { Ipsilateral } \\
\text { Anticipatory } \mathrm{Mu} \\
\text { ERSP }\end{array}$ & - & - & $0.421 * *$ & $0.511 * * *$ & - & - & - & - \\
\hline Anticipatory LI1 & - & - & - & - & $0.378 * *$ & $0.347 *$ & 0.137 & -0.054 \\
\hline Post-stimulus LI & - & - & - & - & -0.047 & -0.127 & -0.184 & -0.047 \\
\hline Flanker & -0.186 & $0.293 *$ & 0.021 & 0.067 & - & - & - & - \\
\hline Card Sort & -0.191 & $0.230^{+}$ & 0.018 & 0.110 & $0.598 * * *$ & - & - & \\
\hline Processing speed & $-0.254^{*}$ & -0.043 & $-0.219+$ & 0.016 & $0.333 * * *$ & $0.421 * * *$ & & - \\
\hline Language & -0.100 & -0.047 & -0.132 & -0.137 & -0.203 & 0.079 & 0.047 & \\
\hline Reaction time & 0.126 & -0.217 & 0.152 & -0.211 & 0.047 & 0.115 & $0.245^{*}$ & -0.092 \\
\hline
\end{tabular}

$+p<0.1, * p<0.05, * * p<0.01, * * * p<0.001$

${ }^{1}$ LI: Mu Lateralization Index 
Mass Univariate Time Frequency

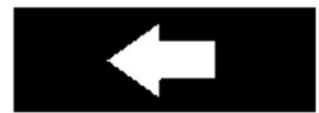

Mass Univariate Scalp Maps

$(-1500$ to $300 \mathrm{~ms}, 5-20 \mathrm{~Hz})$

Contrast: Right vs. Left

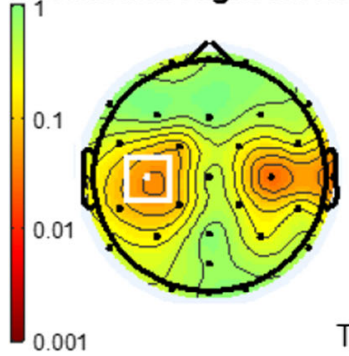

C3

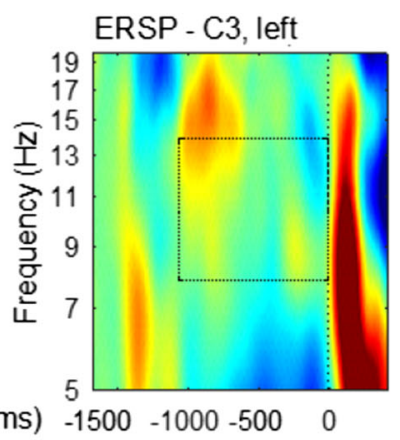

Time (ms) $-1500-1000-500 \quad 0$
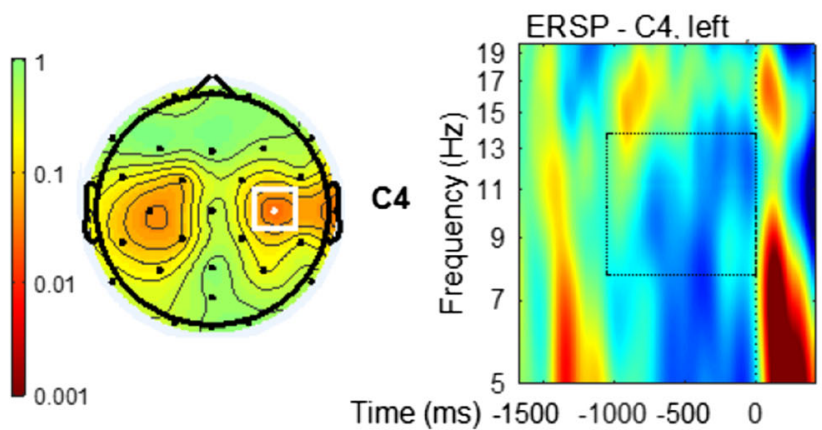

Fig. 2. A priori sample-averaged Mu ERSP. Time-frequency plots display ERSP (event-related spectral perturbation) at left and right central sites $(\mathrm{C} 3 / \mathrm{C} 4)$ across a frequency range of $5-20 \mathrm{~Hz}$ for the time period from $1,500 \mathrm{~ms}$ before the tactile stimulus to $300 \mathrm{~ms}$ after the tactile stimulus. The dashed boxes highlight sample-averaged period (500 to 0

observed. A significant interaction was observed between cue direction and electrode, $\mathrm{F}(1,39)=11.823, p<0.001, \eta^{2 \mathrm{p}}=$ 0.233 . Following stimulation of the left hand, mu ERSP was significantly greater (i.e., the mu rhythm was more synchronized) at the ipsilateral site $\mathrm{C} 3(\mathrm{M}=0.308, \mathrm{SD}=1.337)$ compared with the contralateral site $\mathrm{C} 4(\mathrm{M}=-0.083, \mathrm{SD}=$ $1.555, \mathrm{t}=-3.506, p=0.015, \mathrm{~d}=0.403$ ). Following stimulation of the right hand, mu ERSP was greater at the contralateral site $\mathrm{C} 3(\mathrm{M}=0.393, \mathrm{SD}=1.545)$ compared with ipsilateral site $\mathrm{C} 4(\mathrm{M}=0.079, \mathrm{SD}=1.686, \mathrm{t}=-2.240, p=0.031, \mathrm{~d}=$ $-0.354)$.

\section{Cognitive abilities and anticipatory Mu ERSP}

To examine the relations between task scores and mu ERSP, the conditions used in the previous ANOVAs were collapsed into contralateral (mu ERSP at $\mathrm{C} 3$ for the right hand cue and at $\mathrm{C} 4$ for the left hand cue) and ipsilateral (mu ERSP at $\mathrm{C} 3$ for the left hand cue and at $\mathrm{C} 4$ for the right hand cue) mean $m u$ ERSP values. Pearson correlations were computed among ipsilateral and contralateral mu ERSP in anticipation of (anticipatory) and in response to (post-stimulus) tactile stimulation, and the measures from the NIH Cognitive Toolbox.

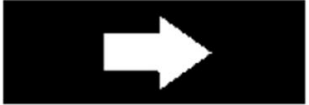

Significance Tests
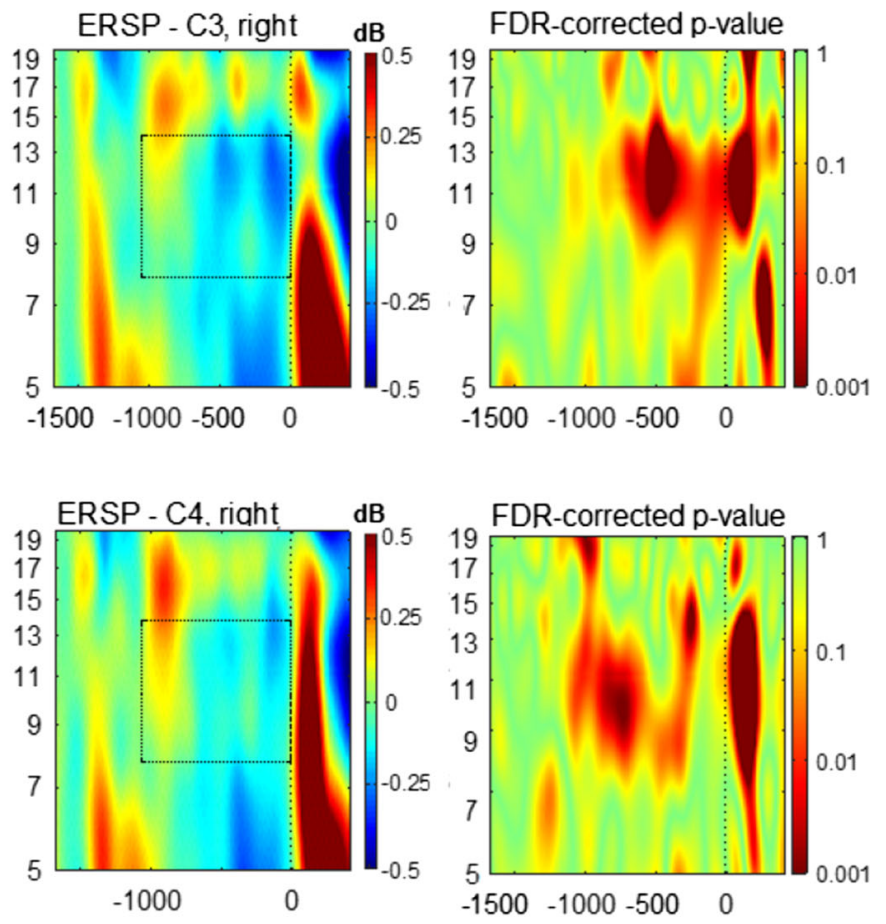

$\mathrm{ms})$ of mu (8-14 Hz) ERSP submitted for subject-specific analysis. The response elicited by the delivery of the tactile stimulus occurs after $0 \mathrm{~ms}$, which was the onset of finger stimulation. The significance test panels show statistical comparisons of ERSP at central electrode sites in response to left vs. right cue

Significant correlations were found between the magnitude of contralateral anticipatory mu ERSP and post-stimulus mu ERSP at both contralateral and ipsilateral sites. Contralateral anticipatory mu ERSP was inversely associated with Processing Speed $(r=-0.254, p=0.02)$, whereas Flanker score was significantly positively associated with ipsilateral anticipatory mu ERSP $(r=0.293, p=0.03)$. Similarly, Card Sort scores were positively associated with ipsilateral anticipatory mu ERSP, but this relation did not reach significance $(\mathrm{r}$ $=0.203, p=0.07)$. Processing Speed ability and task-specific reaction time were significantly correlated $(\mathrm{r}=0.245, p=$ 0.02). As expected, significant positive correlations were observed among the executive function measures and the Processing Speed measure.

To address our hypotheses on the relations between cognitive skills and neural indicators of tactile anticipation, multiple regressions were conducted predicting scores on the Flanker, Card Sort, Receptive Language, and Processing Speed tasks from anticipatory contralateral and ipsilateral mu ERSP. $P$ values were corrected for multiple comparisons using the false discovery rate (FDR). For both Flanker and Card Sort tasks, greater ipsilateral mu ERSP was associated with better EF task performance. Flanker performance was related to ipsilateral mu ERSP, t (39) 
Tactile Anticipation (Pre-Stimulus)

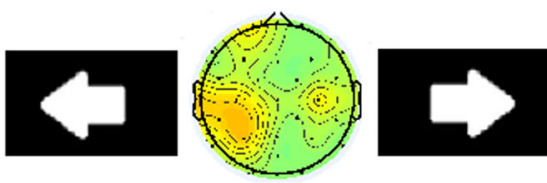

ERSP - left, $-500-0 \mathrm{~ms} \& 8-13 \mathrm{~Hz} \quad$ ERSP - right, $-500-0 \mathrm{~ms} \& 8-13 \mathrm{~Hz}$
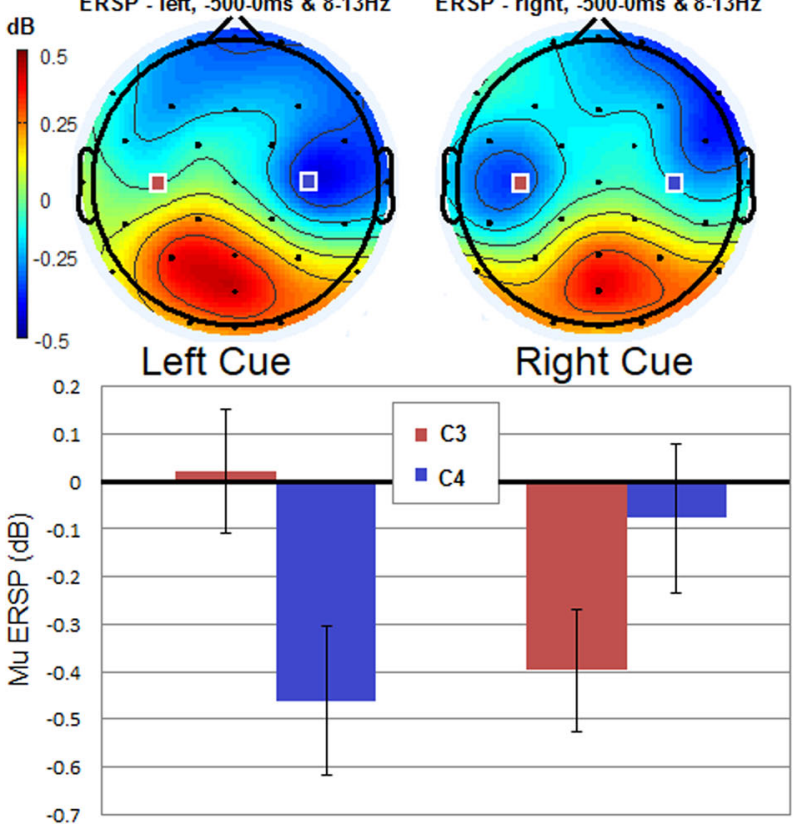

Tactile Response (Post-Stimulus)
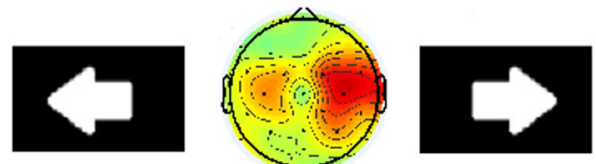

ERSP - left, $20-270 \mathrm{~ms} \& 8-13 \mathrm{~Hz}$

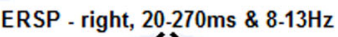

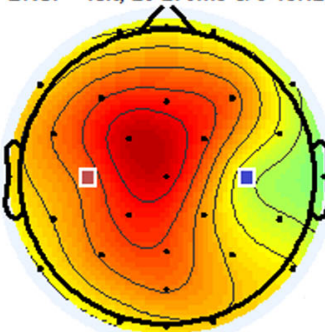

Left Cue

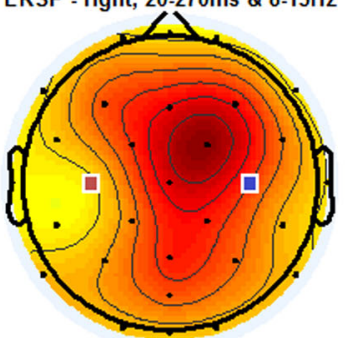

Right Cue

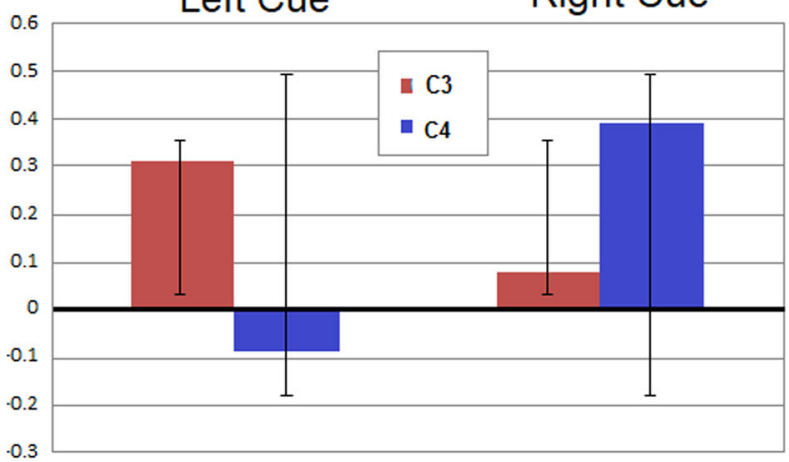

Fig. 3. Alpha-range scalp maps and subject-specific mean Mu amplitude. Scalp maps display mean ERSP for the pre-stimulus anticipation period ( -500 to $0 \mathrm{~ms}$ ) and post-stimulus response period ( $20 \mathrm{~ms}$ to $270 \mathrm{~ms}$ ). Bar graphs display subject-specific mu ERSP at central electrodes C3 and C4 sites

$=2.026, \beta=0.531, p=0.041$, but not with contralateral $\mathrm{mu}$ ERSP. Card Sort performance also was related with ipsilateral mu ERSP, t (39) $=2.219, \beta=0.576, p=0.033$, but was not

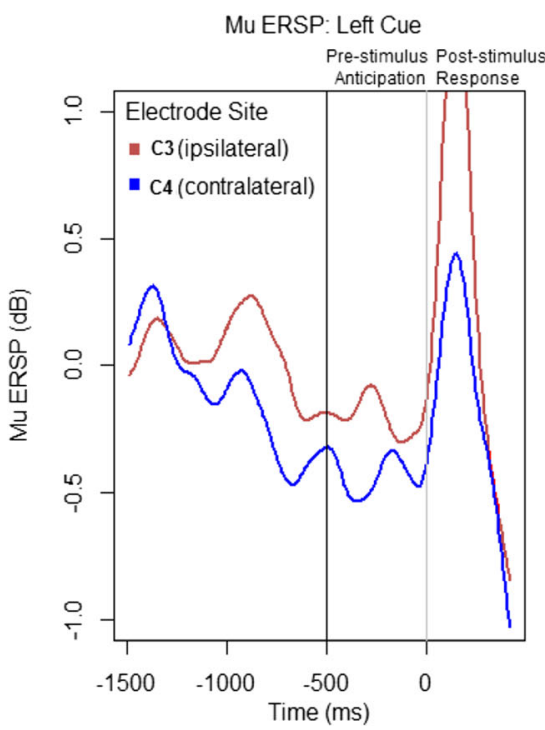

Fig. 4. Continuous ERSP waveforms. Average mu ERSP is plotted within the subject-specific mu frequency range at $\mathrm{C} 3$ and $\mathrm{C} 4$ at during display of the right (red line) or left (blue line) directional cue, plotted from $1,500 \mathrm{~ms}$ prior to tactile stimulus presentation $(0 \mathrm{~ms})$ and to $400 \mathrm{~ms}$ significantly associated with contralateral mu ERSP. Anticipatory Contralateral mu ERSP was related to Processing

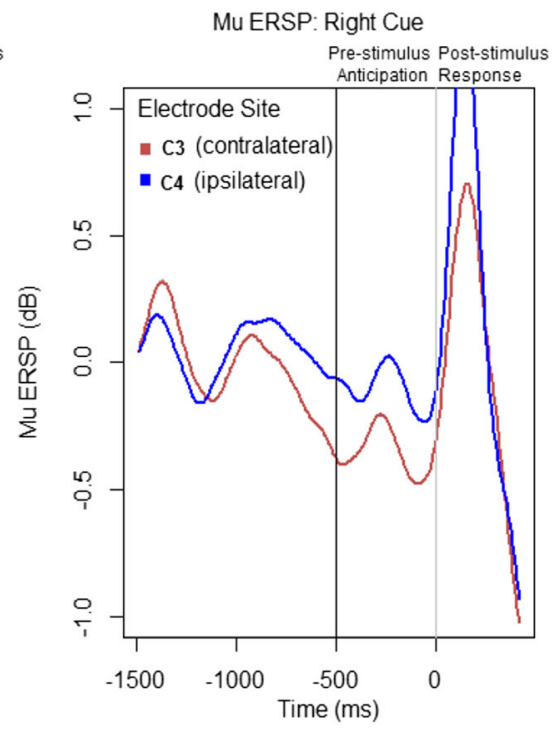

post-stimulus. Pre-stimulus anticipation is averaged from $-500 \mathrm{~ms}$ to 0 $\mathrm{ms}$; post-stimulus response is averaged from $20 \mathrm{~ms}$ to $270 \mathrm{~ms}$. Mu rhythm desynchronization is indicated as negative ERSP values 

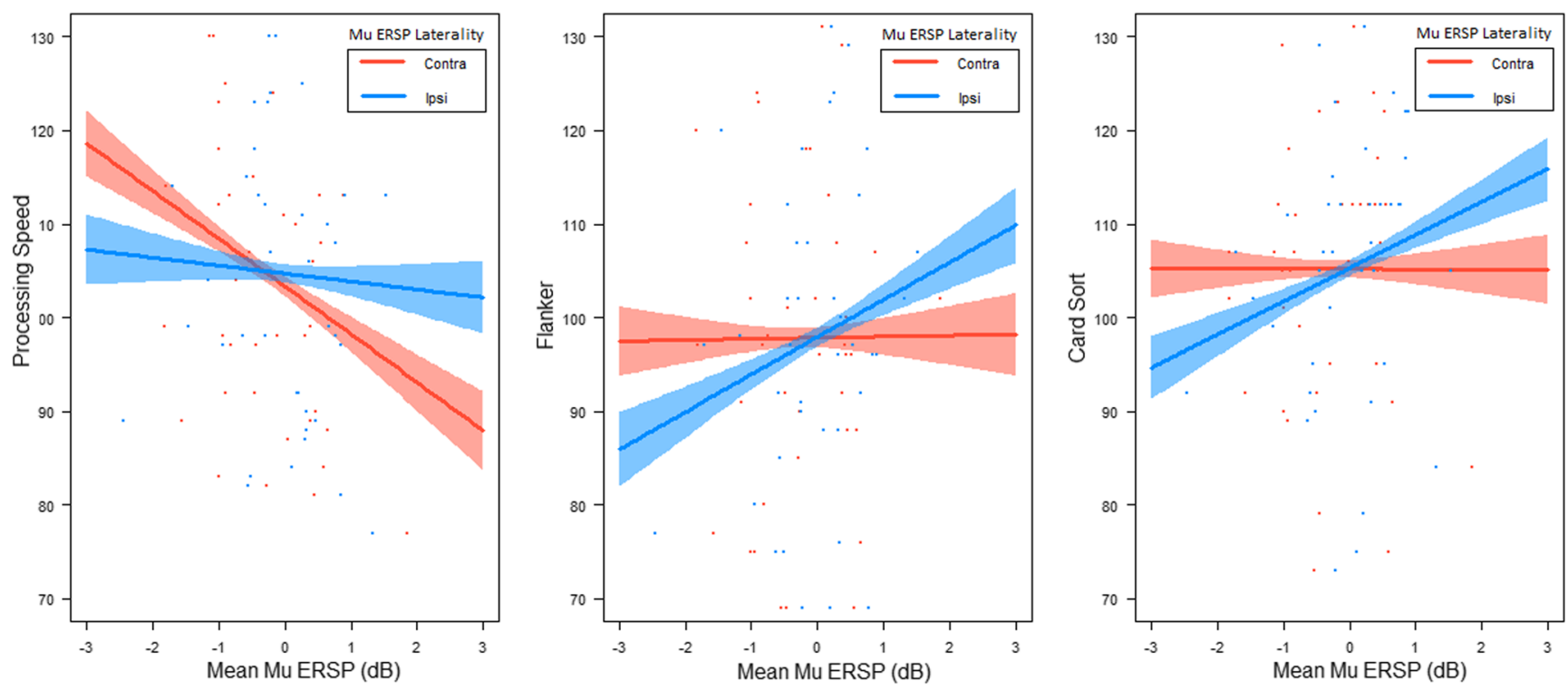

Fig. 5. Linear association of cognitive skills with mean Mu ERSP. The amount of change in mean mu ERSP (relative to 0 change in ERSP) is plotted in units of change in Processing Speed, Flanker, or Card Sort score on the NIH Cognitive Toolbox (Breheny \& Burchett, 2013). The significance of the relation between processing speed and mu laterality is driven solely by anticipatory contralateral mu power; in contrast, the significance of the relation between executive function tasks is driven primarily by variability in subject-specific ipsilateral mu power, above and beyond contralateral mu power

performance on the executive function tasks were similar to the results for mu ERSP at contralateral and ipsilateral sites (Table 4).

\section{Discussion}

This study examined relations between individual differences in sensorimotor mu rhythm activity during anticipation of, and in response to, a target tactile stimulus and variability in cognitive skills, including language, processing speed, and executive function abilities. A visuospatial cue directed participants' attention to the bodily location (right or left hand) of an upcoming tactile stimulus, which they responded to by pressing a foot pedal. Consistent with previous investigations (Jones \& Forster, 2013; Malinowski, 2013; Silas et al., 2019), desynchronization of the sensorimotor mu rhythm was observed in the hemisphere contralateral to the expected location of the tactile stimulus, suggesting that participants directed their attention to the cued hand during the anticipatory epoch (Haegens et al., 2011; Anderson \& Ding, 2011; Van Ede et al., 2014).

The extent of the reduction in mu power observed at contralateral central sites was inversely associated with how rapidly participants compared the similarity of two visual stimuli on a (separate) processing speed task. In turn, performance on the processing speed task was itself related to reaction time in the EEG task, specifically how quickly participants pressed a foot pedal to indicate how many tactile stimuli they perceived. As seen in prior studies, reaction time was not associated with anticipatory changes in pre-stimulus mu power (Haegens et al., 2011; Shen et al., 2018). Instead, more rapid reaction correlation with the anticipatory mu lateralization index $(\mathrm{r}=$ $-0.037, p=0.822$ ). The relations of the lateralization index to 
Table 3 Executive function scores predicted by ipsilateral and contralateral anticipatory (Ant.) Mu ERSP and post-stimulus (PS) Mu

\begin{tabular}{|c|c|c|c|c|c|c|}
\hline Outcome & $\mathrm{B}$ & $\mathrm{SE}$ & $\beta$ & $\mathrm{T}$ & $\mathrm{P}$ & Adj. $R^{2}$ \\
\hline \multicolumn{7}{|l|}{ Flanker } \\
\hline Intercept & 92.315 & 4.173 & & 22.120 & $<0.001 *$ & 0.162 \\
\hline Ant. contralateral Mu & -15.555 & 8.249 & -0.744 & -1.886 & $0.068+$ & \\
\hline Ant. ipsilateral Mu & 14.662 & 6.670 & 0.707 & 2.198 & $0.035 *$ & \\
\hline PS contralateral $\mathrm{Mu}$ & 4.654 & 5.164 & 0.431 & 0.901 & 0.374 & \\
\hline PS ipsilateral Mu & -2.225 & 4.895 & -0.189 & -0.455 & 0.652 & \\
\hline \multicolumn{7}{|l|}{ Card sort } \\
\hline Intercept & 99.742 & 3.472 & & 28.731 & $<0.001 *$ & 0.193 \\
\hline Ant. contralateral Mu & -12.545 & 6.862 & -0.714 & -1.828 & $0.076+$ & \\
\hline Ant. ipsilateral IL Mu & 11.501 & 5.549 & 0.660 & 2.073 & $0.046 *$ & \\
\hline PS contralateral $\mathrm{Mu}$ & 1.504 & 4.296 & 0.166 & 0.350 & 0.728 & \\
\hline PS ipsilateral Mu & 0.988 & 4.072 & 0.100 & 0.243 & 0.810 & \\
\hline \multicolumn{7}{|l|}{ Processing speed } \\
\hline Intercept & 96.075 & 3.836 & & 25.048 & $<0.001 *$ & 0.149 \\
\hline Ant. contralateral Mu & -16.712 & 7.582 & -0.830 & -2.204 & $0.034 *$ & \\
\hline Ant. ipsilateral Mu & 9.079 & 6.131 & 0.455 & 1.481 & 0.148 & \\
\hline PS contralateral $\mathrm{Mu}$ & -0.931 & 4.746 & -0.090 & -0.196 & 0.846 & \\
\hline PS Ipsilateral Mu & 4.736 & 4.499 & 0.417 & 1.053 & 0.300 & \\
\hline \multicolumn{7}{|l|}{ Language } \\
\hline Intercept & 106.101 & 3.319 & & 31.968 & $<0.001 *$ & 0.041 \\
\hline Ant. contralateral Mu & 6.078 & 6.560 & 0.371 & 0.926 & 0.361 & \\
\hline Ant. ipsilateral Mu & -4.074 & 5.305 & -0.250 & -0.768 & 0.448 & \\
\hline PS contralateral $\mathrm{Mu}$ & -7.496 & 4.107 & -0.885 & -1.825 & 0.096 & \\
\hline PS ipsilateral Mu & 5.033 & 3.893 & 0.545 & 1.293 & 0.205 & \\
\hline
\end{tabular}

${ }^{*} p<0.05,{ }^{+} p<0.10$, FDR-corrected for multiple comparisons

Table 4 Cognitive task scores and reaction time by lateralization index of Mu ERSP

\begin{tabular}{lllllll}
\hline Outcome & Mu lateralization index & $\mathrm{B}$ & $\mathrm{SE}$ & $\beta$ & $\mathrm{T}$ & $\mathrm{P}$ \\
\hline Flanker (EF) & (Intercept) & 104.275 & 2.066 & & 50.467 & $<0.001 *$ \\
& Anticipatory & 4.547 & 1.834 & 0.377 & 2.479 & $0.018 *$ \\
& Post-stimulus & -0.333 & 1.324 & -0.038 & -0.251 & 0.803 \\
Card sort (EF) & (Intercept) & 103.692 & 2.221 & & 46.696 & $<0.001 *$ \\
& Anticipatory & 3.284 & 1.423 & 0.352 & 2.307 & $0.027 *$ \\
& Post-stimulus & -1.805 & 1.971 & -0.140 & -0.916 & 0.366 \\
Processing speed & (Intercept) & 96.584 & 2.769 & & 34.879 & $<0.001 *$ \\
& Anticipatory & 2.096 & 1.775 & 0.189 & -1.917 & 0.245 \\
Language & Post-stimulus & -2.204 & 2.458 & -0.143 & -0.897 & 0.376 \\
& (Intercept) & 104.748 & 2.736 & & 38.280 & $<0.001 *$ \\
Reaction time & Anticipatory & -0.558 & 1.754 & -0.052 & -0.318 & 0.752 \\
& Post-stimulus & -0.666 & 2.429 & -0.045 & -0.274 & 0.786 \\
& (Intercept) & 1.521 & 0.027 & & 56.484 & $<0.001 *$ \\
& Anticipatory & -0.006 & 0.017 & -0.049 & -0.322 & 0.749 \\
& Post-Stimulus & 0.056 & 0.024 & 0.357 & 2.322 & 0.005 \\
\end{tabular}

${ }^{*} p<0.05,{ }^{+} p<0.10$, FDR-corrected for multiple comparisons 
times were associated with lateralization of the post-stimulus mu response. The association we report between reaction time and post-stimulus mu lateralization provides further indication that variability in neural responses to somatosensory stimuli may reflect sensory processing speed across modalities, or it could indicate preparation of motor response (Yin et al., 2016). The absence of a significant association between anticipatory changes in mu and time to behavioral response may be attributed to the fact that participants were required to delay the reporting of their responses for $750 \mathrm{~ms}$, until the display instructed them to respond, while previous investigations required an immediate response (Jones et al., 2010; Haegens et al., 2012; Van Ede et al., 2014). Lateralization has been shown to have an impact on preparation for behavioral responses, particularly in predicting reaction time, above and beyond contralateral or ipsilateral mu (or visual alpha) power alone (Van Ede et al., 2014; Haegens et al., 2011; McKinney \& Euler, 2019; Shalev, Van Ede \& Nobre, 2019).

Changes in mu power during anticipation of the tactile stimulus, rather than in response to that stimulus, drove the association of the mu rhythm with performance on the executive function tasks. The tasks tapping executive function included a Flanker task, which required participants to report the direction of a target central visual stimulus in a series, and a Card Sort task, in which participants matched test stimuli on a changing feature (shape or color). Flanker scores were significantly related to increases in anticipatory mu power at central sites ipsilateral to the cued hand, but not to changes at contralateral central sites. Together, changes in mu power accounted for $10 \%$ of variability in flanker task performance. Similarly, scores on the Card Sort task were significantly associated with increases in anticipatory mu power at sites ipsilateral to the cued hand, and were marginally associated with mu desynchronization at central sites contralateral to the cued hand. Together, these changes in mu power accounted for $12 \%$ of variability in Card Sort performance.

These novel findings present evidence for two potential interpretations: it is possible that variability in anticipatory modulation during a simple tactile attention task may reflect individual differences in cognitive skills, while also providing support the conclusion that variability in neural responses specific to sensory anticipation account for variance in measures of cognitive control. Speculatively, we suggest that the ability to anticipate is a plausible developmental building block for executive function (Garon et al., 2008; Nobre \& van Ede, 2018), the development of anticipatory attention as foundational to variability in action and higher-order cognition.

Although studies of the mu rhythm have sometimes only focused on contralateral changes, our findings add further weight to the suggestion that ipsilateral anticipatory mu responses and hemispheric differences in post-stimulus mu modulation are relevant to the coordination of behavioral responses more broadly. The bilateral modulation at central sites following a cue to indicate the anticipated location of a tactile stimulus suggests that the mu rhythm is particularly sensitive to tactile attention (Gomez-Ramirez et al., 2016; Haegens et al., 2012). Work with humans and nonhuman primates suggests that bilateral neural responses in somatosensory cortices may serve to simultaneously managing competing expectancies, reflecting allocation of tactile attention according to goals and providing buffers against potential distraction (Lipton et al., 2006; Haegens et al., 2012; Tamè et al., 2016).

In interpreting the relation of ipsilateral mu activity to executive function, we look to two current explanations for the function of alpha oscillations. In one account, alpha oscillations have been ascribed an inhibitory function (Klimesch et al., 1998), which underpins the notion of a "gating" function of ipsilateral alpha increases. This suggestion is supported by associations between anticipatory ipsilateral alpha power increases and taskrelevant stimulus detection rate as well as speed of behavioral responses, across various sensory modalities (Frey et al., 2015; Van Diepen, Foxe, \& Mazaheri, 2019). In the "active sensing" account of alpha oscillations, increases in power (or power hovering at baseline) over ipsilateral sensory cortices serve to suppress the sampling of events at an unattended location (Schroeder \& Lakatos, 2009; van Ede et al., 2014; Thut et al., 2006). These nonexclusive accounts provide insight into the functional significance of ipsilateral changes in sensory-specific alpha responses (Van Diepen, Foxe, \& Mazaheri, 2019; Liley \& Muthukumarswamy, 2019).

Our results support the suggestion that the variability of anticipatory alpha-range responses in adults may index individual differences in the foundational ability to deploy attention (Shalev, Nobre, \& van Ede, 2019, Jones et al., 2010; Sauseng et al., 2005). It is possible that these results are enhanced for attention in the tactile modality, differences in attention to bodily sensations and/or variability in perceived boundaries between the body, peripersonal space, and extrapersonal space contributed to these differences (Bremner \& Spence, 2017), or that mu oscillations may have greater interindividual variability than other alpha-range rhythms (Coll et al., 2017). Additionally, our findings could be understood as supporting the notion that adults with greater executive function abilities complete the somatosensory anticipation task with a more proactive, deliberate cognitive strategy. This could involve filtering general sensations in a relaxed, passive manner in order to facilitate processing of tactile sensations the cued location, as opposed to actively monitoring the cued bodily location in focused manner (Jones et al., 2010; Van Diepen, Foxe, \& Mazaheri, 2019; Liley \& Muthukumarswamy, 2019).

Prior studies have identified changes in anticipatory mu oscillations during selective attention to the body as an indicator of individual differences in response to mindfulness training (Kerr et al., 2011). Attention to the body is a skill that can be refined and trained over time, perhaps to benefit attention in other modalities (Zeidan et al., 2010). Behavioral 
investigations support the centrality of bodily attention to the improvement of executive function abilities following brief mindfulness training (Mak et al., 2018). A prior fMRI investigation reported the benefits of mindfulness training on participant's ability to resolve conflict during an affective executive function task compared with an active control group, finding variability in the processing of negative stimuli relied on individual differences in neural responses involved in voluntary control of attention (Allen et al., 2012). Further studies can identify whether mechanisms specific focusing on one's own tactile sensations and visceral signals, as opposed to other forms of attention training, may underlie the link between selective attention to the body with broader plasticity of attention-related changes in brain responses (Jha et al., 2007; Tang \& Posner, 2009).

Our findings add to the growing neuroscientific evidence for computational and theoretical frameworks of sensory processing that support a role for anticipatory neural activity in interindividual variability in perceptual experience (Summerfield \& de Lange, 2014). Examining neural responses associated with selective attention to the body provide an opportunity to study multisensory integration (Bremner \& Spence, 2017) in the context of self-models and bodygenerated signals (Allen \& Tsakiris, 2018; Yin et al., 2016). Furthermore, studying anticipation can inform allostatic theories of neural self-regulation and prediction, given that variability in anticipatory neural activity may integrate prior experience with ecologically driven expectancies (Allen \& Friston, 2018; Bruineberg et al., 2018; Jones, 2019). Anticipation appears to elicit a goal-driven calibration of sensory input that may manifest in attention-related shifts in behavior, as well as variable perceptual experiences and stimulus responses (de Lange, Heilbron \& Kok, 2018; Ionta, Gassert, \& Blanke, 2011)

Open Practices Statement The dataset is available at the Open Science Foundation repository: https://osf.io/rb6ud/?view_ only=e7a6141e4b614f3296cc939f7e59fb35

This study was not preregistered.

Acknowledgments The authors thank Yuheiry Rodriguez, Jebediah Taylor, and Olivia Allison for their assistance with data collection. The education of SMW is supported by the NSF Graduate Research Fellowship Program. PJM was partially supported by a grant from NSF (BCS-1460889). Further support for data collection was provided by a CURE grant from the Pennsylvania Department of Health. The authors declare that there is no conflict of interest regarding the publication of this article.

\section{References}

Akshoomoff, N., Beaumont, J. L., Bauer, P. J., Dikmen, S. S., Gershon, R. C., Mungas, D., ... \& Heaton, R. K. (2013). VIII. NIH Toolbox Cognition Battery (CB): composite scores of crystallized, fluid, and overall cognition. Monographs of the Society for Research in Child Development, 78, 119-132.

Allen, M., Dietz, M., Blair, K. S., van Beek, M., Rees, G., VestergaardPoulsen, P., ... Roepstorff, A. (2012). Cognitive-affective neural plasticity following active-controlled mindfulness intervention. J Neurosci, 32, 15601-15610.

Allen, M., \& Tsakiris, M. (2018). The body as first prior: Interoceptive predictive processing and the primacy. Interoceptive predictive processing and the primacy of self-models. The Interoceptive Mind: From Homeostasis to Awareness, 27-40. Oxford University Press, New York, USA

Allen, M., \& Friston, K. J. (2018). From cognitivism to autopoiesis: towards a computational framework for the embodied mind. Synthese, 195, 2459-2482.

Anderson, K. L., \& Ding, M. (2011). Attentional modulation of the somatosensory mu rhythm. Neuroscience, 180, 165-180.

Baumgarten, T. J., Schnitzler, A., \& Lange, J. (2016). Prestimulus alpha power influences tactile temporal perceptual discrimination and confidence in decisions. Cerebral Cortex, 26, 891-903.

Bigdely-Shamlo, N., Mullen, T., Kothe, C., Su, K. M., \& Robbins, K. A. (2015). The PREP pipeline: standardized preprocessing for largescale EEG analysis. Frontiers in Neuroinformatics, 9, 16.

Bremner, A. J., \& Spence, C. (2017). The Development of tactile perception. Adv. in Child Develop., 52, 227-268.

Bremner, A. J., Holmes, N. P., \& Spence, C. (2012). The development of multisensory representations of the body and of the space around the body. Multisensory Develop. 113-136.

Breheny, P., \& Burchett, W. (2013). Visualization of regression models using visreg. R package, $1-9$.

Bruineberg, J., Kiverstein, J., \& Rietveld, E. (2018). The anticipating brain is not a scientist: the free-energy principle from an ecological-enactive perspective. Synthese, 195, 2417-2444.

Buzsaki, G. (2006). Rhythms of the brain. Oxford University Press. New York, NY.

Cheyne, D., Gaetz, W., Garnero, L., Lachaux, J.-P., Ducorps, A., Schwartz, D., \& Varela, F. J. (2003). Neuromagnetic imaging of cortical oscillations accompanying tactile stimulation. Brain Res., $17,599-611$.

Cohen, M. X. (2014). Analyzing neural time series data: theory and practice. MIT Press. Amsterdam, NL.

Coll, M.-P., Press, C., Hobson, H., Catmur, C., \& Bird, G. (2017). Crossmodal classification of mu rhythm activity during action observation and execution suggests specificity to somatosensory features of actions. J Neurosci, 37, 5936-5947.

Corbetta, M., Kincade, J. M., Ollinger, J. M., McAvoy, M. P., \& Shulman, G. L. (2000). Voluntary orienting is dissociated from target detection in human posterior parietal cortex. Nature Neuroscience, 3, 292-297.

Cuevas, K., Cannon, E. N., Yoo, K., \& Fox, N. A. (2014). The infant EEG mu rhythm: methodological considerations and best practices. Developmental Review, 34, 26-43.

Delorme A \& Makeig S. (2004). EEGLAB: an open source toolbox for analysis of single-trial EEG dynamics including independent component analysis. J Neurosci Methods, 134, 921.

de Lange, F. P., Heilbron, M., \& Kok, P. (2018). How do expectations shape perception? Trends Cogn. Sci, 22, 764-779.

Diamond, A. (2013). Executive Functions. Annual Review of Psychology, 64, 135-168.

Engel, A. K., Fries, P., \& Singer, W. (2001). Dynamic predictions: Oscillations and synchrony in top-down processing. Nature Reviews Neuro., 2, 704-710.

Frey, J. N., Ruhnau, P., \& Weisz, N. (2015). Not so different after all: The same oscillatory processes support different types of attention. Brain Res., 1626, 183-197. 
Foxe, J. J., \& Snyder, A. C. (2011). The role of alpha-band brain oscillations as a sensory suppression mechanism during selective attention. Front. Psyc., 2, 154-160.

Garon, N., Bryson, S. E., \& Smith, I. M. (2008). Executive function in preschoolers: a review using an integrative framework. Psychol. Bull., 134, 31-45.

Gazzaley, A., \& Nobre, A. C. (2012). Top-down modulation: bridging selective attention and working memory. Trends Cogn. Sci,, 16, 129-135.

Gomez-Ramirez, M., Hysaj, K., \& Niebur, E. (2016). Neuralmechanisms of selective attention in the somatosensory system. J. Neurophys., $116,1218-1231$.

Haegens, S., Händel, B. F., \& Jensen, O. (2011). Top-down controlled alpha band activity in somatosensory areas determines behavioral performance in a discrimination task. J Neurosci, 31, 5197-5204.

Haegens, S., Luther, L.,\& Jensen, O. (2012). Somatosensory anticipatory alpha activity increases to suppress distracting input. J. Cogn. Neurosci, 24, 677-685.

Haegens, S., Cousijn, H., Wallis, G., Harrison, P. J., \& Nobre, A. C. (2014). Inter-and intra-individual variability in alpha peak frequency. Neuroimage, 92, 46-55.

Hoffmann, S., \& Falkenstein, M. (2008). The correction of eye blink artefacts in the EEG: a comparison of two prominent methods. PLoS One, 3(8), e3004.

Haggard, P., Taylor-Clarke, M., \& Kennett, S. (2003). Tactile perception, cortical representation and the bodily self. Current Biology, 13, R170-R173.

Holmes, N. P., \& Spence, C. (2004). The body schema and the multisensory representation(s) of peripersonal space. Cog. Process, 5, 94 105

Howell, D. C. (2016). Fundamental statistics for the behavioral sciences. Nelson Education.

Ionta, S., Gassert, R., \& Blanke, O. (2011). Multi-sensory and sensorimotor foundation of bodily self-consciousness-an interdisciplinary approach. Frontiers in Psych., 2, 383-396.

Jones, S. R., Kerr, C. E., Wan, Q., Pritchett, D. L., Hämäläinen, M., \& Moore, C. I. (2010). Cued spatial attention drives functionally relevant modulation of themu rhythm in primary somatosensory cortex. J Neurosci, 30, 13760-13765.

Jones, A., \& Forster, B. (2013). Independent effects of endogenous and exogenous attention in touch. Somatosens Mot Res., 30, 161-166.

Jones, A. (2019). Temporal expectancies and rhythmic cueing in touch: The influence of spatial attention. Cognition, 182, 140-150.

Jha, A. P., Krompinger, J., \& Baime, M. J. (2007). Mindfulness training modifies subsystems of attention. Cogn Affect Behav Neurosci, 7, 109-119.

Kerr, C. E., Jones, S. R., Wan, Q., Pritchett, D. L., Wasserman, R. H., Wexler, A., ... Littenberg, R. (2011). Effects of mindfulness meditation training on anticipatory alpha modulation in primary somatosensory cortex. Brain Res. Bull., 85, 96-103.

Klimesch, W. (1999) EEG alpha and theta oscillations reflect cognitive and memory performance: a review and analysis. Brain Research Reviews, 29, 169-195.

Klimesch, W., Doppelmayr, M., Russegger, H., Pachinger, T., \& Schwaiger, J. (1998). Induced alpha band power changes in the human EEG and attention. Neuro. Letters, 244, 73-76.

Klimesch, W., Sauseng, P., \& Hanslmayr, S. (2007). EEG alpha oscillations: The inhibition-timing hypothesis. Brain Res. Reviews, 53, 63-88.

Liley, D. T., \& Muthukumarswamy, S. D. (2019). Evidence that alpha blocking is due to increases in system-level oscillatory damping not neuronal population desynchronisation. bioRxiv, 729723.

Linkenkaer-Hansen, K., Nikulin, V. V., Matias Palva, J., Kaila, K., \& Ilmoniemi, R. J. (2004). Stimulus-induced change in long-range temporal correlations and scaling behaviour of sensorimotor oscillations. Eur J Neurosci, 9, 203-211.
Lipton, M. L., Fu, K.-M. G., Branch, C. A., \& Schroeder, C. E. (2006). Ipsilateral hand input to area $3 \mathrm{~b}$ revealed by converging hemodynamic and electrophysiological analyses in macaque monkeys. J Neurosci, 26, 180-185.

Longo, M. R., Azañón, E., \& Haggard, P. (2010). More than skin deep: body representation beyond primary somatosensory cortex. Neuropsychologia, 48, 655-668.

Lopes da Silva, F. (2013). EEG and MEG: relevance to neuroscience. Neuron, 80, 1112-1128.

Mak, C., Whittingham, K., Cunnington, R., \& Boyd, R. N. (2018). Efficacy of mindfulness-based interventions for attention and executive function in children and adolescents - A systematic review. Mindfulness, 9, 59-78.

Malinowski, P. (2013). Neural mechanisms of attentional control in mindfulness meditation. Frontiers in Neurosci., 7, 8-22.

Mazaheri, A., Nieuwenhuis, I. L. C., van Dijk, H., \& Jensen, O. (2009). Prestimulus alpha and mu activity predicts failure to inhibit motor responses. Hum Brain Mapp, 30, 1791-1800.

McKinney, T. L., \& Euler, M. J. (2019). Neural anticipatory mechanisms predict faster reaction times and higher fluid intelligence. Psychophysiology, 56, e13426.

McKinney, T. L., Euler, M. J., \& Butner, J. E. (2020). It's about time: The role of temporal variability in improving assessment of executive functioning. The Clinical Neuropsychologist, 34, 619-642.

Miyake, A., \& Friedman, N. P. (2012). The nature and organization of individual differences in executive functions: Four general conclusions. Current Directions in Psychological Science, 21, 8-14.

Murphy, J. W., Foxe, J. J., \& Molholm, S. (2016). Neuro-oscillatory mechanisms of intersensory selective attention and task switching in school-aged children, adolescents and young adults. Developmental Science, 18, 9-18.

Myers, N. E., Stokes, M. G., Walther, L., \& Nobre, A. C. (2014). Oscillatory brain state predicts variability in working memory. J Neurosci., 34, 7735-7743.

Nigg, J. T. (2017). Annual Research Review: On the relations among self-regulation, self-control, executive functioning, effortful control, cognitive control, impulsivity, risk-taking, and inhibition for developmental psychopathology. Journal of Child Psychology and Psychiatry, 58, 361-383.

Nobre, A. C., \& van Ede, F. (2018). Anticipated moments: temporal structure in attention. Nature Reviews Neurosci, 19, 34

Oldfield, R. C. (1971). The assessment and analysis of handedness: the Edinburgh inventory. Neuropsychol., 9, 97-113.

Penfield, W., \& Boldrey, E. (1937). Somatic motor and sensory representation in the cerebral cortex of man as studied by electrical stimulation. Brain, 60, 389-443.

Pfurtscheller, G. (1989). Functional topography during sensorimotor activation studied with event-related desynchronization mapping. J Clinical Neurophys., 6, 75-84.

Posner, M. I. (1980). Orienting of attention. Quarterly J Exp. Psych., 32, $3-25$.

Ricciardi, E., Bonino, D., Gentili, C., Sani, L., Pietrini, P., \& Vecchi, T. (2006). Neural correlates of spatial working memory in humans: a functional magnetic resonance imaging study comparing visual and tactile processes. J Neurosci., 139, 339-349.

Rungratsameetaweeman, N., Itthipuripat, S., \& Serences, J. T. (2019). Dissociable effects of attention and expectation on perceptual decision making. Journal of Vision, 19, 49b.

Sauseng, P., Klimesch, W., Stadler, W., Schabus, M., Doppelmayr, M., Hanslmayr, S., Gruber, W.R., \& Birbaumer, N. (2005). A shift of visual spatial attention is selectively associated with human EEG alpha activity. Eur J Neurosci, 22, 2917-2926.

Sadaghiani, S., \& Kleinschmidt, A. (2016). Brain networks and $\alpha$-oscillations: Structural and functional foundations of cognitive control. Trends in Cog. Neurosci., 20, 805-817. 
Samaha, J., \& Postle, B. R. (2015). The speed of Alpha-band oscillations predicts the temporal resolution of visual perception. Current Bio., $30,113-120$.

Silas, J., Tipple, A., \& Jones, A. (2019). Event-related alpha desynchronization in touch-comparing attention and perception. Neurosci. Letters, 131-137.

Shalev, N., Nobre, A. C., \& van Ede, F. (2019). Time for What? Breaking Down Temporal Anticipation. Trends in Neurosciences, 42(6), 373374.

Serino, A. (2019). Peripersonal space (PPS) as a multisensory interface between the individual and the environment, defining the space of the self. Neurosci \& Biobehav Rev., 99, 138-159.

Schroeder, C. E., \& Lakatos, P. (2009). Low-frequency neuronal oscillations as instruments of sensory selection. Trends in Hum. Neurosci., $32,9-18$.

Shen, G., Smyk, N. J., Meltzoff, A. N., \& Marshall, P. J. (2018). Using somatosensory mismatch responses as a window into somatotopic processing of tactile stimulation. Psychophys., 19, 110-120.

Spitzer, B., \& Blankenburg, F. (2011). Stimulus-dependent EEG activity reflects internal updating of tactile working memory in humans. Proc Natl Acad Sci., 108, 8444-8449.

Summerfield, C., \& De Lange, F. P. (2014). Expectation in perceptual decision-making: neural and computational mechanisms. Nature Reviews Neurosci., 15, 745-750.

Tamè, L., Braun, C., Holmes, N. P., Farnè, A., \& Pavani, F. (2016). Bilateral representations of touch in the primary somatosensory cortex. Cog. Neuropsyc., 33, 48-66.

Tamè, L., Azañón, E., \& Longo, M. R. (2019). A conceptual model of tactile processing across body features of size, shape, side, and spatial location. Frontiers in Psychology, 10, 291.

Tang, Y. Y., \& Posner, M. I. (2009). Attention training and attention state training. Trends in Cog. Sci., 13, 222-227.

Thut, G., Nietzel, A., Brandt, S. A., \& Pascual-Leone, A. (2006). $\alpha$-band electroencephalographic activity over occipital cortex indexes visuospatial attention bias and predicts visual target detection. J Neurosci, 26, 9494-9502.

Van der Lubbe, R. H., \& Utzerath, C. (2013). Lateralized power spectra of the EEG as an index of visuospatial attention. Advances in Cognitive Psychology, 9, 184.

van Ede, F., de Lange, F. P., \& Maris, E. (2012). Attentional cues affect accuracy and reaction time via different cognitive and neural processes. J Neurosci., 32, 10408-10412.

van Ede, F., Jensen, O., \& Maris, E. (2010). Tactile expectation modulates pre-stimulus beta-band oscillations in human sensorimotor cortex. Neuroimage, 51, 867-876. van Ede, F., Szebényi, S., \&Maris, E. (2014). Attentional modulations of somatosensory alpha, beta and gamma oscillations dissociate between anticipation and stimulus processing. Neuroimage, 97, 134 141.

van Diepen, R. M., Cohen, M. X., Denys, D., \& Mazaheri, A. (2015). Attention and temporal expectations modulate power, not phase, of ongoing alpha oscillations. J. Cogn. Neurosci., 27, 1573-1586.

van Diepen, R., Foxe, J. J., \& Mazaheri, A. (2019). The functional role of alpha-band activity in attentional processing: The current zeitgeist and future outlook. Current Opinion in Psych., 29, 229-238.

Weiss, S.M., Meltzoff, A. N., \& Marshall, P. J. (2018). Neural measures of anticipatory bodily attention in children: Relations with executive function. Dev Cogn Neurosci., 34, 148-158.

Willoughby, M. T., Blair, C. B., Kuhn, L. J., \& Magnus, B. E. (2018). The benefits of adding a brief measure of simple reaction time to the assessment of executive function skills in early childhood. J Exp. Child Psyc., 170, 30-44.

Wittenberg, M. A., Baumgarten, T. J., Schnitzler, A., \& Lange, J. (2018). U-shaped relation between prestimulus alpha-band and poststimulus gamma-band power in temporal tactile perception in the human somatosensory cortex. Journal of Cognitive Neuroscience, 30, $552-564$.

Yin, S., Liu, Y., \& Ding, M. (2016). Amplitude of sensorimotor mu rhythm is correlated with BOLD from multiple brain regions: A simultaneous EEG-fMRI study. Front Hum Neurosci., 10, 364-380.

Zhang, Y., \& Ding, M. (2010). Detection of a weak somatosensory stimulus: role of the prestimulus mu rhythm and its top-down modulation. Journal of Cognitive Neuroscience, 22, 307-322.

Zanto, T. P., \& Gazzaley, A. (2009). Neural suppression of irrelevant information underlies optimal working memory performance. J Neurosci., 29, 3059-3066.

Zeidan, F., Johnson, S. K., Diamond, B. J., David, Z., \& Goolkasian, P. (2010). Mindfulness meditation improves cognition: Evidence of brief mental training. Consc. \& Cog., 19, 597-605.

Zelazo, P. D. (2006). The Dimensional Change Card Sort (DCCS): A method of assessing executive function in children. Nature prot. 1, 297-306.

Zelazo, P. D., Anderson, J. E., Richler, J., Wallner-Allen, K., Beaumont, J. L., \& Weintraub, S. (2013). II. NIH Toolbox Cognition Battery (CB): Measuring executive function and attention. SRCD Monographs, 78, 16-33.

Publisher's note Springer Nature remains neutral with regard to jurisdictional claims in published maps and institutional affiliations. 